\title{
Les substances hallucinogènes provenant de l'ergot de seigle et des volubilis
}

\section{Hallucinogen alcaloid derivatives from Ergot and Morning Glory}

Marie-Hélène GHYSEL ${ }^{(1) *}$, Francis TROTIN ${ }^{(2)}$,

(1) Laboratoire de Police Scientifique, 7, boulevard Vauban - F-59000 LILLE

(2) Laboratoire de Pharmacognosie, Faculté de Pharmacie, rue du Professeur Laguesse - BP 83 - F-59006 LILLE

* Auteur à qui adresser la correspondance : Marie-Hélène GHYSEL, Laboratoire de Police Scientifique, 7, boulevard Vauban - F-59000 LILLE - Tél : 0320128979 - Fax : 0320128999

\section{$R E ́ S U M E ́$}

Parmi les substances hallucinogènes, les dérivés ergotés occupent une place non négligeable. Différents champignons ou plantes en renferment. Le plus connu est l'ergot de seigle, un sclérote parasite du seigle. Dans les cas d'intoxications par des céréales contaminées les victimes ont présenté de nombreux troubles dont des hallucinations, mais il n'a pas été décrit de cas d'individus consommant sciemment de l'ergot de seigle pour avoir des hallucinations. En 1943, Albert Hofmann qui travaillait sur les composés de ce champignon et notamment sur l'acide lysergique a synthétisé de nombreux produits dont le diéthylamide de l'acide lysergique (LSD). Ce produit est tellement actif qu'il est déposé sous forme d'une goutte sur un support tel que du papier buvard qui sera placé sous la langue. Ces doses renferment généralement de 50 à $100 \mu \mathrm{g}$ de LSD. Assez rapidement apparaissent des troubles des perceptions sensorielles et somesthésiques. Ces troubles sont imprévisibles.

Le LSD est rapidement transformé par $N$-déméthylation, $N$ dééthylation et hydroxylation. En fonction des appareils utilisés pour le détecter et de la dose absorbée, le LSD ou ses

(Reçu le 16 janvier 2004 ; accepté le 6 février 2004)

\section{SUMMARY}

Among natural hallucinogens, ergot derivatives are very important. The best known source is the Ascomycete Claviceps purpurea, ergot, a rhizomorph that parasitizes the grain of rye and other grasses. In 1943, Albert Hofmann synthetised a derivative of lysergic acid, which he called LSD 25.50 to $100 \mu \mathrm{g}$ of this very powerfull substance are enough to induce hallucinations and disturb the sensitive and somesthetic perceptions. LSD is rapidly metabolised by $N$ demethylation, $N$-deethylation and hydroxylation. LSD or its metabolites, according to the technical means, could be detected in urine during 30 to 120 hours. At present time, 2oxo-3-hydroxy-LSD, seems most interesting due to its higher concentration and longer presence in urine.

Among the family Convolvulaceae, Ololiuqui, Rivea corymbosa and previously introduced ornamental climbing plants as Ipomoea violacea (Morning glory) are presented. This volubilis contained ergine, product displaying psychodysleptic properties for $2 \mathrm{mg}$ doses, the effects varying according to the number of seeds consumed. 
métabolites séraient détectables dans les urines pendant 30 à 120 heures. Le 2-oxo-3-hydroxy-LSD, présent en concentration plus importante est actuellement celui qui permet de détecter plus longtemps une concentration antérieure de LSD. Des alcaloïdes indoliques voisins de ceux de l'ergot de seigle ont été retrouvés dans des Convolvulacées, l'ololiuqui, Rivea corymbosa et des liserons, Ipomoea violacea. Cette dernière plante, originaire du Mexique, appelée "Morning glory» par les Anglo-saxons, a été introduite en Europe comme plante ornementale. Ces volubilis renfermeraient de l'ergine, substance responsable des effets psychodysleptiques à la dose de $2 \mathrm{mg}$. Les effets sont variables selon le nombre de graines absorbées.

\section{MOTS-CLÉS}

Ergot, LSD, ergine, Ololiuqui, Rivea corymbosa, Ipomoea violacea, Morning glory, intoxication, analyses.

\section{Introduction}

L'ergot de seigle Claviceps purpurea est un sclérote parasite du seigle renfermant des alcalö̈des indoliques, il est à l'origine d'une maladie assez spectaculaire appelée ergotisme ou encore «Feu de Saint-Antoine», «Feu Sacré», «Mal des Ardents», noms divers donnés à des épidémies dues à l'ingestion, le plus souvent en temps de disette, de farines contaminées par lui. La dernière en France n'est pas si éloignée et aurait eu lieu vers les années 1950, à Pont Saint Esprit dans le Gard (cependant, certains disent qu'il s'agirait plutôt d'une intoxication mercurielle). Les symptômes de ces intoxications sont : maux de ventre, convulsions, hallucinations, gangrènes des membres, brûlures internes, élévation ou, au contraire, baisse de la tension artérielle.

En 1943 Albert Hofmann qui travaillait sur les composés de ce champignon et notamment sur l'acide lysergique a synthétisé de nombreux produit dont le $25^{\text {ine }}$ d'une série de dérivés amidés, connu sous le nom de LSD 25, qui est le diéthylamide de l'acide lysergique. C'est surtout ce dérivé à propriétés hallucinogènes qui sera évoqué ici.

Des dérivés de l'ergine sont également présents dans d'autres plantes comme l'Ipomoea violacea, Rivea corymbosa, Argyreia nervosa.

\section{L'ergot de seigle et le LSD Description}

L'ergot de seigle, Claviceps purpurea (Fries) Tulasne, est un champignon du groupe des Ascomycètes, qui parasite le seigle, mais aussi le froment et l'orge. Il s'agit de la forme résistante du champignon qui a un cycle de reproduction complexe. L'ergot se présente sous la forme d'une excroissance - le sclérote - qui se

\section{KEY-WORDS}

Ergot, LSD, ergine, Ololiuqui, Rivea corymbosa, Ipomoea violacea, Morning glory, intoxication, analysis.

fixe au niveau des caryopses (grains de la céréale). De forme plus ou moins arquée, il mesure de 1 à 4 centimètres de long sur 3 à 8 millimètres de large, il est de couleur pourpre foncé et vire au noir lorsqu'il est à maturité, (photographie 1).

\section{Composition}

Elle est fort complexe. On retrouve notamment des lipides, des stérols, des glucides et des amines. Les substances pharmacologiquement actives sont des alcaloïdes ergoliniques. L'ergoline est le système tétracyclique fondamental de la quinzaine d'alcaloïdes isolés. D'après leur constitution et leur solubilité dans l'eau on répartit ces alcaloïdes en deux groupes principaux :

- les alcaloïdes à chaîne polypeptidique, insolubles dans l'eau, le groupe de l'ergotamine et le groupe de l'ergotoxine,

- les alcaloïdes non polypeptidiques, dont les bases sont solubles dans l'eau comme l'ergométrine (1).

Nous nous intéresserons ici à un dérivé semi-synthétique réalisé par Albert Hofmann à partir de l'acide lysergique : le LSD 25 ou diéthyl amide de l'acide lysergique, (figure 1).

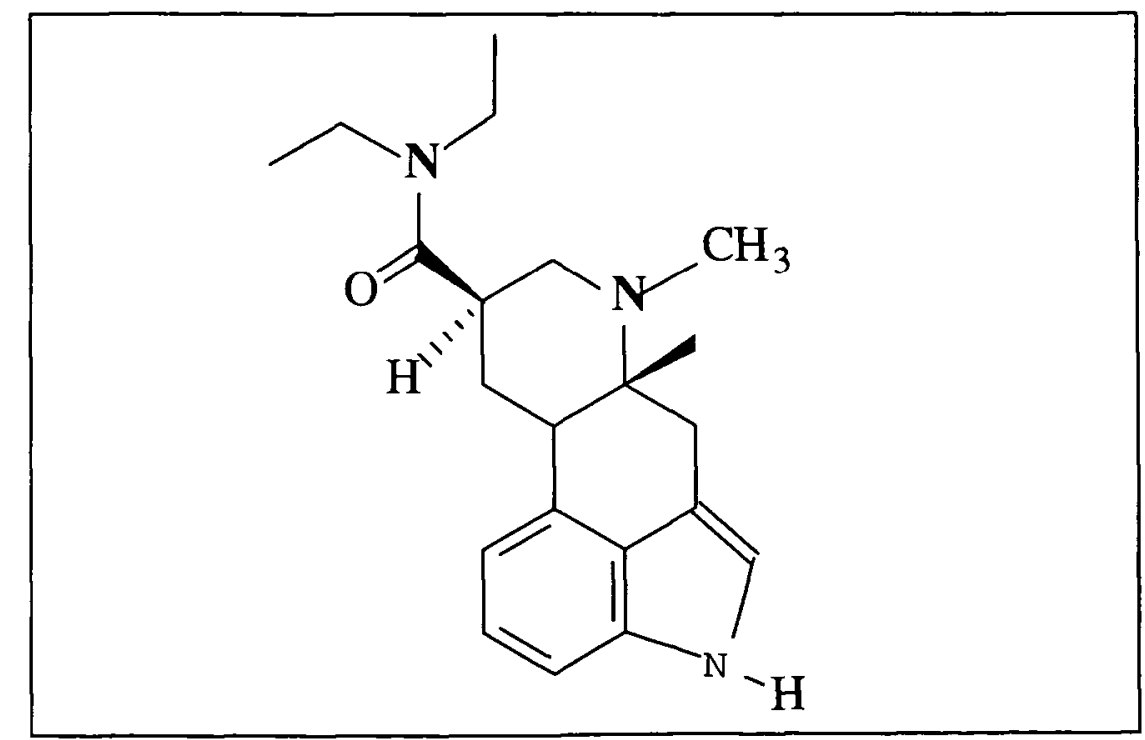

Figure 1 : Formule chimique du LSD. 


\section{Historique}

Il fut fabriqué pour la première fois en 1938 par Stoll et Hofmann, chimistes suisses qui travaillaient pour le laboratoire Sandoz. À partir de l'acide lysergique, ils synthétisèrent le diéthylamide, connu sous le nom de LSD-25. Après sa synthèse, ils le laissèrent de côté et continuèrent leurs recherches sur d'autres plantes.

En 1943, A. Hofmann obtient l'autorisation de poursuivre ses recherches sur cette molécule. C'est au cours de sa synthèse qu'il est pris de malaise, rentre chez lui et est pris de visions extraordinaires. Son état s'améliore au bout de deux heures. Persuadé que le LSD est à l'origine de son trouble, il décide de tester sur lui sa découverte, absorbe $0,25 \mathrm{mg}$ de tartrate de LSD et attend que les effets se manifestent. Moins de trois quarts d'heures plus tard, les premiers symptômes apparaissent. Il se fait raccompagner chez lui par son assistante, les effets de la drogue ne s'estomperont que tard dans la soirée. A Hofmann parle en ces termes de son expérience...

«Mes vertiges et mes sensations de faiblesse prenaient de telles proportions par moments que je ne pouvais même plus me tenir debout : il me fallut $m$ 'allonger sur le canapé. A ce moment là, mon environnement s'était transformé de façon angoissante. Toutes les choses se mouvaient dans l'espace, les objets familiers, le mobilier prenaient des formes grotesques, menaçantes la plupart du temps. Elles étaient comme animées d'un mouvement perpétuel, comme emplies d'une angoisse intérieure. C'est à peine si je reconnus ma voisine qui m'apportait du lait, [...] ce n'était plus Mme R., c'était une sorcière malfaisante, perfide, qui cachait derrière son fard, un visage diabolique. Mais il y eut plus grave encore que ces modifications grotesques du monde extérieur : les transformations que je ressentis en moimême, à l'intérieur de mon être. Tous mes efforts de volonté pour contenir cet éclatement du monde extérieur et cette dissolution de mon moi me paraissaient voués à l'échec. Un démon avait pénétré en moi, il avait pris possession de mon corps, de mes sens et de mon âme. Je sautai, je criai pour m'en débarrasser, mais finalement, je retombai épuisé sur le canapé. La substance que j'avais voulue expérimenter avait eu raison de moi. Elle était ce démon sarcastique qui triomphait de ma volonté. Une angoisse horrible me prit d'être devenu fou. J'avais débarqué sur un autre monde où les notions de temps et d'espace étaient différentes. Mon corps me paraissait insensible, inerte, étranger. Etais-je dans la mort? Etait-ce le passage dans l'au-delà ? Par moments j'avais l'impression d'être en dehors de mon corps ; et dans ces moments là, comme observateur extérieur, je prenais conscience de tout le tragique de ma situation. [...] Lentement, enfin je revenais d'un monde étrange, inquiétant, dans la réalité quotidienne familière. [...] C'est alors que je commençai à jouir du spectacle inouï de formes et de couleurs, qui durait encore derrière mes yeux fermés. Aussi changeantes que dans un kaléidoscope, des images multicolores, fantastiques arrivaient sur moi, s'ouvraient en cercles ou en spirales, puis se refermaient, telles des fontaines de couleurs jaillissantes, s'ordonnaient et se croisaient, en un flot ininterrompu. Le plus étonnant, c'est que toutes les perceptions acoustiques, le bruit d'une poignée de porte, d'une voiture qui passait dans la rue, se transformaient en sensations optiques. Chaque son nouveau produisait une image aux formes et aux couleurs nouvelles». (D'après A. Hofmann, Le LSD mon enfant terrible).

Le LSD venait d'entrer dans l'histoire. Il fut bien sûr expérimenté chez l'animal et chez l'homme par les Laboratoires Sandoz, et de nombreux dérivés furent synthétisés, dont aucun n'avait de propriétés aussi nettes sur le psychisme. Les analyses entreprises sur le LSD ne seront publiées qu'après la guerre, de crainte que les nazis n'en fassent un usage militaire. Par la suite, des expérimentations à visée médicale furent effectuées mais au début des années soixante, le LSD est vite devenu une drogue recherchée.

\section{Préparation du principe actif}

L'ergot de culture obtenu par infestation artificielle de champs de seigle avec des variétés d'ergots très riches en alcaloïdes fut longtemps la première source d'ergolines. Pour éviter de contaminer les champs voisins de céréales alimentaires, on prenait soin d'utiliser pour l'ergot des variétés de seigle tardives ou un hybride du blé et du seigle (triticale) arrivant à maturité après la récolte des céréales destinées à la consommation.

Depuis plusieurs dizaines d'années, on est capable de synthétiser artificiellement, par voie chimique, certains alcaloïdes et leurs dérivés qui présentent un intérêt en thérapeutique, mais on a surtout recours à des substances d'origine naturelle, obtenues par fermentation industrielle, que l'on transforme chimiquement en dérivés utilisables en thérapeutique.

Actuellement, dans les laboratoires clandestins le LSD serait synthétisé à partir de l'acide lysergique selon plusieurs méthodes. Les impuretés peuvent être éliminées par chromatographie, ou par extraction par des solvants organiques. Le LSD base est instable. C'est généralement le tartrate qui est synthétisé.

\section{Propriétés physico-chimiques}

Noms chimiques : 9,10-Didéhydro-N,N-diéthyl-6méthylergoline-8-béta-carboxamide; N,N-diéthyl-Dlysergamide; d-lysergic acid diéthylamide; Noms commerciaux : Lysergide, d-lysergic acid diéthylamide, 
LSD, LSD-25, lysergsäure diethylamid ; Appellations : acide, LSD, buvard, cubes, battery acid, blooter acid, blue heaven, ...

Numéro de CAS : 50-37-3 ; Formule brute : $\mathrm{C}_{20} \mathrm{H}_{25} \mathrm{~N}_{3} \mathrm{O}$ Poids moléculaire : 323,44 . Sous forme de tartrate : 860,9

Le LSD peut avoir trois autres formes spatiales, soit l'iso-LSD, le L-LSD et le L-iso-LSD. Il est très sensible à l'air et à la lumière. Au contact de l'oxygène de l'air, il est décomposé par oxydation et en présence de lumière, il se transforme en produit inactif.

\section{Présentation du LSD}

Le LSD est un produit de couleur blanche qui se présente sous forme de cristaux sans odeur et sans goût. Son point de fusion se situe entre 80 et $85^{\circ} \mathrm{C}$. Le point de fusion du tartrate se situe entre 198 et $200^{\circ} \mathrm{C}$. Le sel est soluble dans l'eau et l'alcool. Il donne une solution incolore, inodore. Pour être vendu aux toxicomanes, i1 est fréquemment déposé sur des supports de papier buvard (cf. photographie $\mathrm{n}^{\circ} 2$ ). Plus rarement, il peut être déposé sur des petits comprimés (2) ou mélangé à de l'amphétamine. Dans ce dernier cas, il s'agissait de petits carrés de papier de $8 \mathrm{~mm}$ de côté, à l'effigie d'Elvis Presley (3).

\section{Utilisation}

Parce ce que la structure chimique du LSD a des similarités avec certains neuromédiateurs et que ses effets s'apparentent à certains aspects de la psychose, il constitue une matière intéressante pour la recherche dans le domaine de la psychologie. Cette drogue a été utilisée en recherche, entre autre pour l'étude du cerveau et de ses processus biochimiques sous-tendant les fonctions psychiques. Une de ses propriétés est son effet bloquant de la sérotonine. Il a également été utilisé en psychiatrie. En psychothérapie des doses de 100 à $750 \mu \mathrm{g}$ ont été données (4). Au début des années soixante, de la consommation de cette drogue chez des artistes est né l'art psychédélique.

Cette substance est essentiellement maintenant utilisée illicitement par des toxicomanes.

Elle est classée dans la catégorie des produits stupéfiants. En France, la baisse des saisies de LSD amorcée en 2001 s'est poursuivie en 2002. 20691 doses avaient été saisies par les services de police et de gendarmerie en $2000 ; 6718$ en 2001 et 4262 doses en 2002.30 usagers de LSD avaient été interpellés en 2002 contre 72 en 2001. 14 trafiquants de LSD ont été interpellés en 2002 (5). En Belgique une enquête réalisée en 1998 a montré que le LSD aurait été utilisé par $4 \%$ des jeunes bruxellois. Ce serait en Grande-Bretagne que la prévalence d'utilisation chez les jeunes de 15 à 16 ans serait la plus importante au niveau européen : $12 \%$ (6).

\section{Effets}

L'isomère dextrogyre du LSD serait l'hallucinogène le plus puissant connu à ce jour. Les effets sont imprévisibles, variables selon les individus et la quantité consommée. Ils peuvent être différenciés en plusieurs phases

- Le départ : les premiers signes apparaissent 20 à 40 min après la prise par voie orale. Les symptômes physiques généralement décrits sont : mydriase, sueurs, tachycardie, tremblements, hyperthermie, hypertension. Ils peuvent durer 2 heures.

- Le «voyage» ou «trip» des Anglo-saxons. La sensation peut être agréable (good trip) avec euphorie et bien-être; ou désagréable (bäd trip) avec sensations de persécution, folie, dislocation, angoisse... Il se manifeste par des modifications

- des perceptions sensorielles (visions kaléidoscopiques colorées, confusion des perceptions (on verra une odeur ou un son ...)),

- des perceptions somesthésiques (étrangeté du monde extérieur, impression de flottaison dans l'espace, difficulté d'apprécier le temps écoulé....),

- de l'humeur (euphorie, angoisse, anxiété...), puissante émotivité, conscience altérée.

Cette phase peut durer 8 à 12 heures, voire beaucoup plus. Si le sujet fait un «mauvais voyage», il faut alors impérativement le rassurer et l'accompagner. Le sujet sous LSD est très influençable, l'environnement où il se trouve et les conditions de prise de la drogue sont très importants, ainsi que sa personnalité. La tournure agréable ou désagréable du voyage est imprévisible.

- Le «retour». Le sujet reprend contact avec son monde environnant, il reste très fatigué pendant 1 à 2 jours.

On note également des "retours d'acide" ou "flashbacks" qui consistent en une réapparition brève mais désagréable de l'expérience hallucinatoire, en l'absence d'une nouvelle prise de LSD. Ils peuvent survenir n'importe quand.

Cette drogue est 100 fois plus forte que la psilocybine et 4000 fois plus forte que la mescaline. Elle n'entraîne pas de dépendance ni de tolérance. Cependant, elle peut provoquer de l'anxiété, des dépressions, voire des troubles psychiques durables.

Le risque majeur au cours de la prise de LSD est en fait lié à l'état psychique du sujet, avec accès suicidaire ou folie meurtrière. Le patient doit être maîtrisé rapidement et placé sous surveillance, il peut être nécessaire d'administrer un neuroleptique inhibiteur comme l'halopéridol à forte dose. 


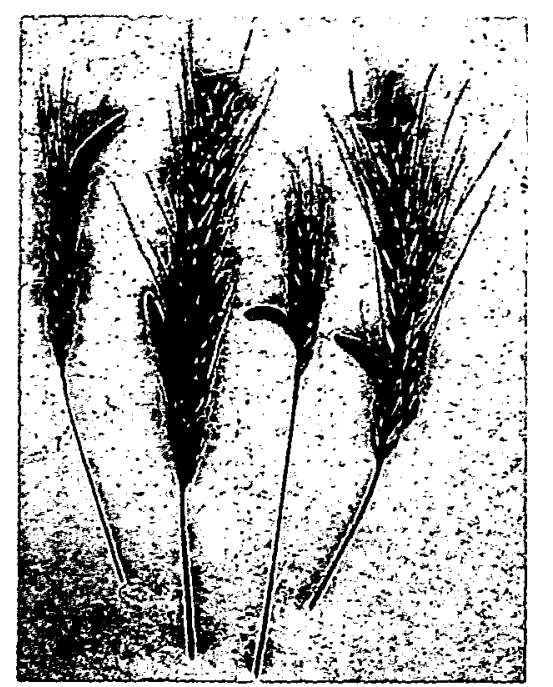

Photographie 1 : Épi de seigle avec ergot d'après http://www.wisc.edu/botany/fungi/oct99.html

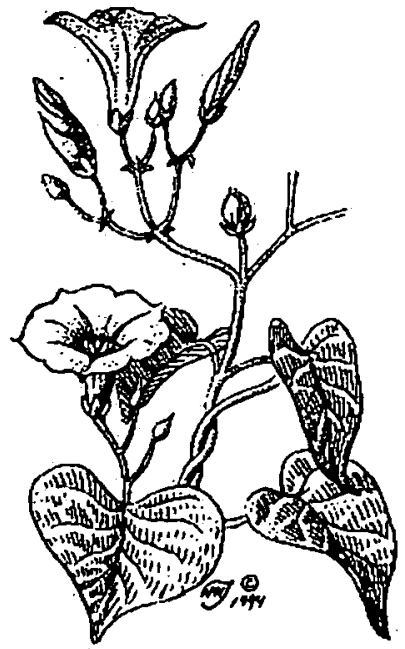

Photographie 3 : Reproduction de Rivea corymbosa d'après "psychedelic shamanism" sur le site internet Erowid.org
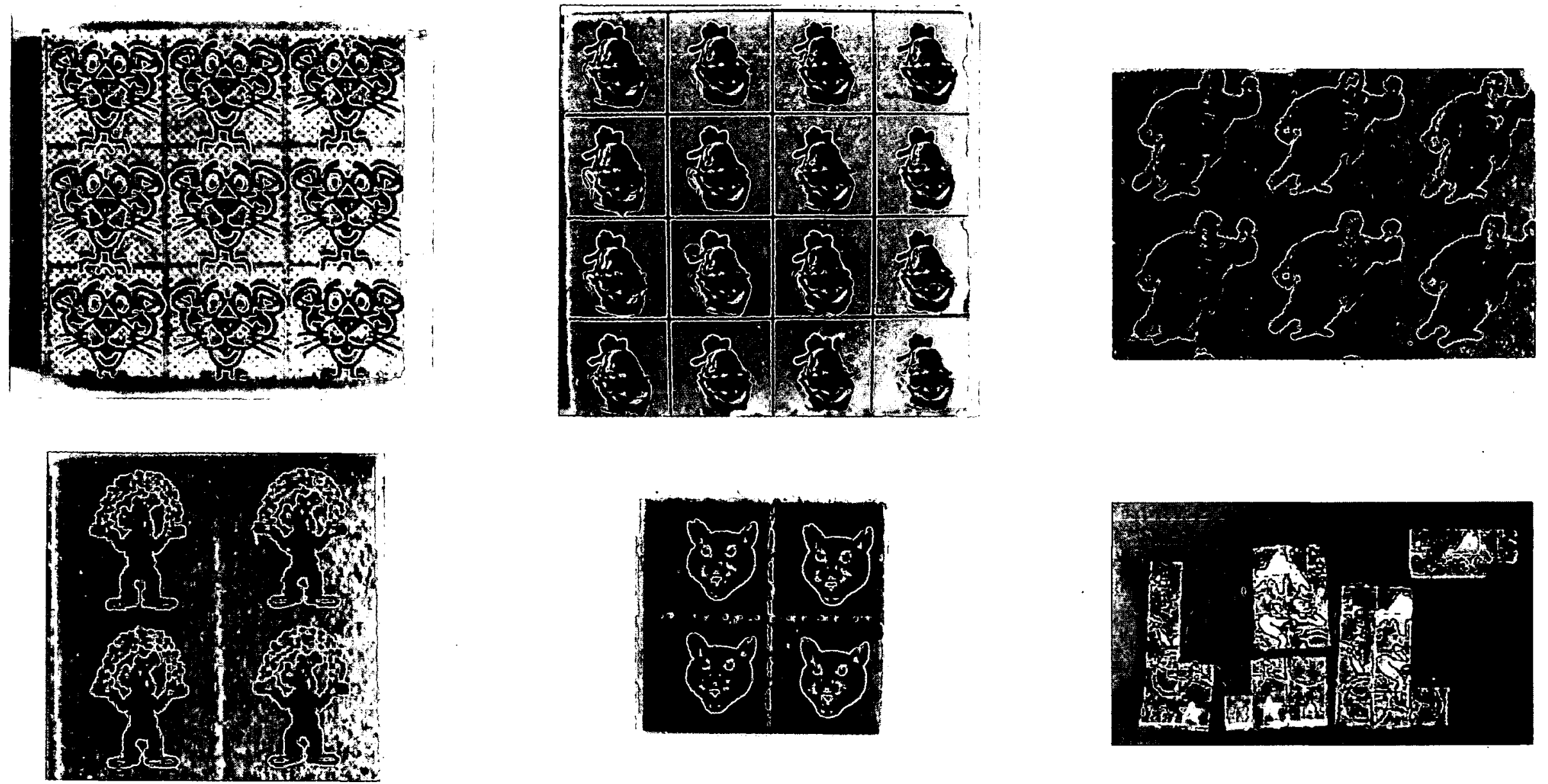

Photographie 2 : Doses de LSD (photographie LPS).

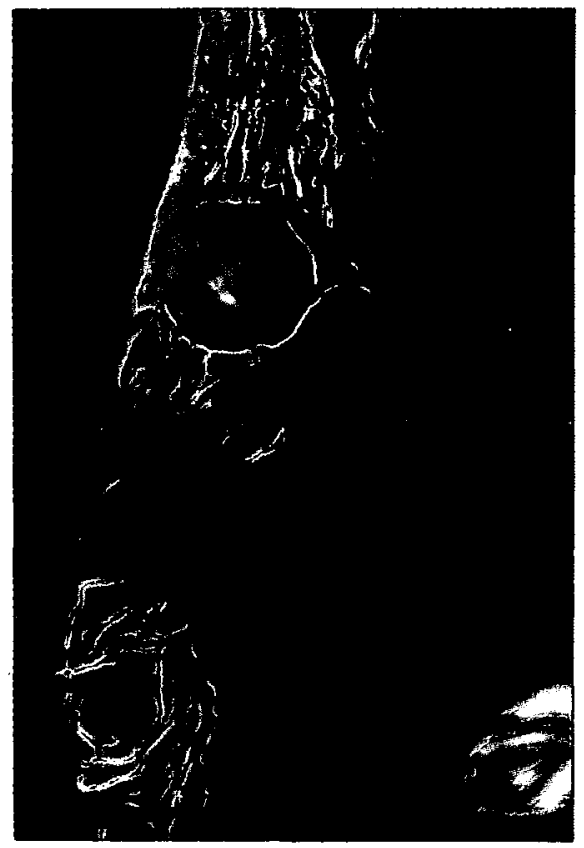

Photographie 4 : Ipomoea violacea photographie prise au jardin botanique de Lille en 1988 (36).

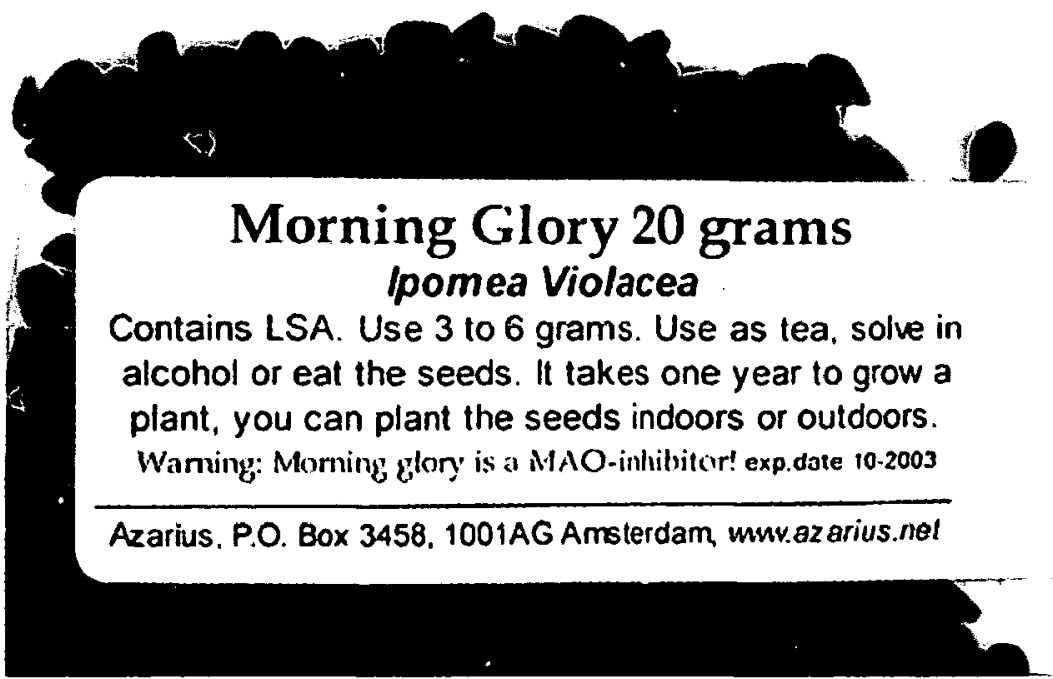

Photographie 5 : Échantillon de graines de Morning glory (photographie LPS). 


\section{Pharmacocinétique}

La voie d'administration la plus courante est la voie sublinguale. Comme il s'agit d'un produit qui a été rapidement classé dans les stupéfiants et qui peut donner des réactions imprévisibles, peu d'études ont été effectuées sur l'homme. On sait qu'il est fortement lié aux protéines plasmatiques, qu'il est rapidement absorbé au niveau gastro-intestinal et qu'il subit une transformation hépatique importante. Sa demi-vie serait comprise entre 2 et 5 heures. Il a un volume de distribution de $0,3 \mathrm{~L} / \mathrm{Kg}$, la fixation aux protéines plasmatiques est de $90 \%$. $(4,7)$

Dans les urines, seul $1 \%$ du LSD est retrouvé inchangé en $24 \mathrm{H}$. Les métabolites connus sont le déméthylLSD, (1,2\%), le 13 et le 14-hydroxy-LSD sous forme glucuroconjuguées ( 2 à $3 \%$ ), le deséthyl-LSD $(1 \%)$ et le 2-oxo-3-hydroxy-LSD. Le métabolite majeur correspondrait à un noyau aromatique portant une fonction amine primaire $(8,9)$.

Des essais réalisés dans une clinique suisse de psychothérapie sur 2 volontaires ayant reçu du LSD à la dose de $4 \mu \mathrm{g} / \mathrm{kg}$ ont montré que le métabolite 2-oxo-3hydroxy-LSD pouvait être détecté par CLHP-SM/SM pendant 96 heures alors que le LSD était sous la limite de détection $(10 \mathrm{pg} / \mathrm{ml})$ en $48 \mathrm{~h}$ pour 1 patient et en 72 $\mathrm{h}$ pour l'autre.(10)

Par méthode radioimmunologique, le LSD, ou ses métabolites qui réagissent avec le test, ont été détectés dans les urines pendant 34 à 120 heures chez 7 personnes ayant reçu $300 \mu \mathrm{g}$ de LSD par voie orale (11).

\section{Mécanisme d'action}

L'action pharmacologique des alcaloïdes de l'ergot trouve son origine dans l'analogie structurale qu'ils présentent avec les amines biogènes (12). Dès 1954 Wooley fait un lien entre la structure des hallucinogènes indoliques et la sérotonine. En 1994, Markel montre 1' affinité des hallucinogènes indoliques pour les récepteurs du type 5-HT1A et 5-HT2A. Le LSD produirait ses effets par fixation et activation des récepteurs 5-hydroxytryptamine 2(5-HT2). Il influence également les fonctions neurophysiologiques liées à la dopamine. Cependant le mécanisme d'action psychomimétique est à notre connaissance encore inconnu $(8,12)$.

\section{Toxicité et complications}

Quelques cas de convulsions ou de coma ont été signalés, cependant les complications sont davantage d'ordre psychiatrique. Des crises d'angoisse et de panique aiguë, des troubles durables du type dépression ou sentiments de persécution sont décrits.

En racontant leur «voyage», même plusieurs mois plus tard, de l'angoisse ou de la panique peuvent réapparaître.

\section{Analyse \\ 1/Le produit}

Le LSD est souvent extrait par du méthanol. Une des meilleures méthodes pour des concentrations de l'ordre de 30 à $150 \mu$ g consiste à placer la dose dans $5 \mathrm{ml}$ d'un mélange méthanol/eau (1/1) pendant 20 minutes dans un bain d'ultrasons à $20^{\circ} \mathrm{C}$ (13).

Les maxima des spectres ultra-violets ont de $315 \mathrm{~nm}$ en solution acide et 310 en solution alcaline. Les maxima du spectre infra-rouge sont : 1626, 1307, 1136, 1066, $1212,749 \mathrm{~cm}^{-1}$, et les fragments $\mathrm{m} / \mathrm{z}$ caractéristiques du spectre de masse sont : 323, 221, 181, 222, 207, 72, 223, 324.

\section{Les tests de coloration,}

Le paradiméthylaminobenzaldéhyde et le test d'Ehrlich colorent le LSD en bleu, le test de Mandelin et le test de Marquis donnent une coloration grise $(4,14)$. Ces résultats doivent être confirmés par une méthode de référence.

\section{La CLHP}

De nombreuses techniques ont été publiées $(13,14)$. Citons à titre indicatif celle réalisée à partir d'une colonne en silice Spherisorb S5W ; utilisant un mélange éluant composé de $1,17 \mathrm{~g}(0,01 \mathrm{M})$ de perchlorate d'ammonium dans 11 de méthanol, amené à $\mathrm{pH}$ 6,7 par addition de $1 \mathrm{ml}$ d'hydroxyde de sodium $0,1 \mathrm{M}$ dans du méthanol. La détection se fait par UV à $313 \mathrm{~nm}$ ou en fluorescence par excitation à $308 \mathrm{~nm}$ et émission de 370 à $700 \mathrm{~nm}(13)$.

\section{La CPG}

Là aussi, de nombreuses conditions analytiques sont envisageables. Par exemple, une séparation sur colonne HP5 MS 30m, 0,25mm/ 0,25 $\mu$, et une détection par spectrométrie de masse en impact électronique.

\section{L'infra-rouge}

Une analyse par infra-rouge à transformée de Fourier peut être réalisée après examen des échantillons au microscope (15).

\section{2/Les milieux biologiques}

Le dosage du LSD est difficile à réaliser du fait des concentrations très faibles dans les liquides biologiques. En effet les doses absorbées sont généralement de l'ordre de 50 à $250 \mu \mathrm{g}$, le métabolisme est intense puisque seulement $1 \%$ du LSD se retrouve inchangé dans les urines.

Le LSD est instable en solution acide et le $\mathrm{pH}$ alcalin peut favoriser sa conversion en son diasteréo-isomère inactif l'iso-LSD $(8,16)$. La tendance actuelle est de 
rechercher le métabolite 2-oxo-3-hydroxy-LSD dans les urines, car dans une étude sur 39 échantillons, il a été montré que les concentrations de ce métabolite étaient en moyenne 12,8 fois plus importantes que celle du LSD(10).

\section{Les prélèvements}

- Le sang. Le LSD ne serait détectable dans le sang que quelques heures ( $6 \mathrm{~h}$ environ)

- Les urines. C'est le prélèvement le plus analysé pour rechercher une consommation. Le LSD est sensible aux ultra-violets et à la chaleur, mais sa concentration dans l'urine est à peu prés stable pendant 1 mois. Il en reste $37 \%$ dans un échantillon conservé à température ambiante à la lumière pendant 222 jours et $63 \%$ si conservé au réfrigérateur (17). Il est également nécessaire de vérifier l'adultération de l'urine en vérifiant le $\mathrm{pH}$. Un pH compris entre 5 et 8,5 n'influe pas sur la valeur du dosage (18).

- Les cheveux. Du LSD a été détecté à l'état de traces chez 2 utilisateurs chroniques sur 17 (19).

\section{Méthodes immunologiques}

Différentes techniques ont été mises au point et commercialisées : RIA, CEDIA, ELISA, EMIT, KIMS, Abuscreen OnLine, ou micro-plaques EIA. Malheureusement, ces tests croisent peu avec le métabolite 2-oxo-3-hydroxy-LSD, qui est présent en plus grande quantité dans les urines (10). Le taux de faux positifs est important.

L'EMIT® II de chez Dade Behring dont le seuil de positivité est fixé à $0,5 \mathrm{ng} / \mathrm{ml}$, s'adapte sur les automates Cobas Mira ${ }^{\circledR}$, SYVA 30 R® et Hitachi $717 \AA$ (8). Mais le taux de faux positifs est élevé $(3,7 \%)$. La fluoxétine, la chlorpromazine, certains médicaments antiémétiques et cardiovasculaires donneraient des résultats faussement positifs (20). Sur 48 échantillons d'urine positifs avec le test CEDIA DAU LSD assay, seulement 13 ont été confirmés par CLHP avec détection par fluorescence après extraction par immunoaffinité. Une comparaison réalisée avec le test DPC Coata-Count RIA donne des résultats comparables pour des concentrations inférieures à un seuil de $500 \mathrm{pg} / \mathrm{ml}$. Au moins une quinzaine de substances positivent le test CEDIA DAU LSD. Il s'agit de : ambroxol, prilocaïne, pipampérone, diphenhydramine, métoclopramine, amitriptyline, doxépine, atracurium, bupivacaïne, doxylamine, lidocaïne, mépivacaïne, prométhazine, ranitidine et tramadol (21).

\section{L'extraction}

Des extractions liquide/liquide ou en phase solide ont été décrites. Le $\mathrm{pH}$ optimal d'extraction est compris entre 8,6 et 9,2. L'évaporation d'une solution méthanolique acidifiée de LSD sous azote pouvant provoquer des pertes, une neutralisation préalable est préférable (19).

Des extractions spécifiques peuvent être réalisées soit avec des colonnes adaptées comme par exemple la Cerex ${ }^{\circledR}$ Polychrom CLIN II 5mg, 5ml (SPEware San Pedro CA) reliée à un dispositif Speedisk® (J.T. Baker, Phillipsburg, NJ).(Le coefficient d'extraction du métabolite oxo par cette technique est de $92 \%$ (22)), soit les disques SPEC.PLUS MP1 (3ml, 30mg, Ansys, Irvine, CA, USA), ou encore par immunoaffinité avec le LSD ImmunElute extraction kit (microgenics corp Fremont CA). L'ajout d'éthylène glycol à la fin de la préparation de l'échantillon recouvre la surface du verre et permet d'éviter des pertes (23). Du LSD marqué au deutérium LSD- $_{3}$ ou LSD-D 10 peut-être utilisé comme étalon interne.

De nombreuses méthodes de recherches et de dosage du LSD dans les liquides biologiques ont été décrites dans l'article réalisé par Françoise Vincent (8), notamment :

La chromatographie couche mince haute performance $8 \mathrm{ml}$ d'urine sont extraits par un mélange d'éther de pétrole, de dichlorométhane et d'alcool isoamylique en présence d'ammoniaque. Cette technique permet de détecter jusqu'à $0,4 \mathrm{ng} / \mathrm{ml}$ de LSD (24).

La Chromatographie Liquide Haute Performance (CLHP)

Les méthodes sont nombreuses (8). La détection peut se faire par fluorescence (longueur d'onde d'excitation $315 \mathrm{~nm}$, émission $420 \mathrm{~nm}$ ), avec une limite de détection de $0,05 \mathrm{ng} / \mathrm{ml}(21,23)$ ou par détecteur de masse. Des méthodes en CLHP-SM (22), CLHP-ES-SM (25), CLHP-SM/SM (26) ont été décrites. Par CLHP-ES-SM la limite de quantification pour le LSD, l'iso-LSD et le nor-LSD est de $0,05 \mathrm{ng} / \mathrm{ml}$, pour le nor-iso-LSD elle est de $0,1 \mathrm{ng} / \mathrm{ml}(25)$.

\section{La Chromatographie en Phase Gazeuse (CPG)}

couplée à différents types de détecteurs.

Là aussi, les méthodes sont nombreuses(8). Le LSD étant peu volatil et facilement absorbable, une dérivation est préconisée. Les dérivés les plus couramment formés sont les dérivés triméthyl silylés (TMS) ou trifluoroacétylés (TFA). Les fragments $\mathrm{m} / \mathrm{z}$ principaux du 2-oxo-3-hydroxy-LSD-bis-TMS sont 309 et 499. La limite de détection du lysergide et de ses métabolites dans les urines est variable selon les techniques, de 29 $\mathrm{ng} / \mathrm{ml}$ à $10 \mathrm{ng} / \mathrm{ml}$ par GC-MS-MS $(27,28)$.

\section{Concentrations sanguines-cas médico-légaux}

Une dose de $160 \mu \mathrm{g}$ a été administrée à 16 personnes, les concentrations plasmatiques mesurées à intervalle régulier varient de 0 à $9 \mu \mathrm{g} / \mathrm{L}$ pendant 8 heures après la 


\section{prise (7).}

200 à $400 \mu \mathrm{g}$ ont été administrés à 8 personnes, les concentrations de lysergide ou de ses métabolites dans l'urine sont comprises entre 1 et $55 \mu \mathrm{g} / \mathrm{L}$ dans les $24 \mathrm{H}$ après l'ingestion (4). Des consommateurs ont survécu à l'absorption de plus de $10 \mathrm{mg}$. 8 personnes pensant «sniffer» de la cocaïne ont en fait consommé du tartrate de LSD. Ils ont présenté des vomissements et des signes d'hyperactivité sympathique : hyperthermie, coma, arrêt respiratoire mais ils ont tous pu être réanimés. Les concentrations de tartrate de LSD dans le sérum étaient comprises entre 2,1 et $26 \mu \mathrm{g} / \mathrm{L}$, et dans le contenu gastrique de 1000 à $7000 \mu \mathrm{g} / 100 \mathrm{~mL}$ (29). Des concentrations plasmatiques supérieures à $1 \mu \mathrm{g} / \mathrm{ml}$ seraient toxiques (4).

A notre connaissance peu de cas de décès imputables au LSD ont été décrits. Griggs et coll. en 1977 trouvent par spectrofluorimétrie $0,312 \mathrm{mg} / \mathrm{dL}$ dans le foie (30), Fysh et coll. en 1985 rapporte le cas d'un jeune homme décédé $16 \mathrm{~h}$ après son arrivée à l'hôpital, les concentrations ante mortem sont de $14,8 \mathrm{ng} / \mathrm{ml}$ par RIA et 8 ng/ml par CLHP (31). Cependant ces résultats sont à prendre avec prudence, en effet Smith et coll. (32) ont montré que selon la méthode utilisée des erreurs pouvaient être possibles soit par fluorescence naturelle de l'urine ou du plasma, soit par dosage concomitant des métabolites.

La toxicité du LSD réside davantage dans les modifications du comportement qu'il peut provoquer. Des conduites suicidaires, des accidents, des automutilations,... ont été décrits et peuvent avoir des conséquences fatales.

\section{Les Volubilis}

Des alcaloïdes indoliques voisins de ceux de l'ergot de seigle ont été retrouvés dans des Convolvulacées, notamment l'ololiuqui, Rivea corymbosa ou Turbina corymbosa; Ipomoea violacea, le «morning glory» des anglo-saxons ainsi que Argyreia nervosa.

\section{Historique}

L'ololiuqui, utilisée au Mexique à l'époque préhispanique, a été ensuite consommée de façon occulte après la christianisation. Cette plante, employée à des fins magiques et religieuses, est décrite dans les chroniques des premiers espagnols arrivés au Mexique peu après la découverte par Cortez (33-36).

\section{Description}

La plus ancienne description de Rivea corymbosa revient à Francisco Hernandez, envoyé par Philippe II d'Espagne pour étudier la flore et la faune mexicaine. Il décrit : «l'ololiuqui...est une plante volubile aux feuilles cordiformes, vertes et minces, dont les tiges vertes et graciles vont en s'amincissant vers le haut qui porte des fleurs blanches à corolle allongée. La graine est ronde et ressemble beaucoup à la coriandre» (photographie 3).

En 1941 Schultes décrit Rivea corymbosa, encore nommée Ipomoea sidaefolia, : «la plante est grande, grimpante, forestière, à l'allure de liserons du genre Convolvulus (de convolvere en latin qui veut dire «autour de son support») (35). Les feuilles sont longues de 5 à $9 \mathrm{~cm}$ et larges de 2,5 à $4 \mathrm{~cm}$, généralement cordiformes ou ovalaires, entières, glabres ou très modérément pubescentes, longuement pétiolées. Le pédoncule est axillaire, la plupart du temps très fleuri. Les fleurs naissent en cyme condensée. La corolle est gamopétale, infundibuliforme ou hypocratériforme, de 2 à $4 \mathrm{~cm}$ de long, blanches ou blanchâtres à lobes entiers et glabres. Elle a 2 stigmates, étamines incluses. L'ovaire est glabre, à deux cellules. Les sépales sont ovales à lancéolés, hypertrophiés au niveau du fruit anguleux, assez ligneux, d'environ $1 \mathrm{~cm}$ de long. Le fruit est elliptique, bacciforme, indéhiscent, il possède une graine.

Ipomoea violacea (photographie 4), parfois employée avec ou à la place de Rivea corymbosa présente des différences significatives

- les sépales sont membraneux et souvent foliacés

- les fleurs sont rarement hypocratériformes

- la capsule est déhiscente avec 2 graines anguleuses allongées et noires.

Ipomoea violacea, appelée «Morning glory» par les anglo-saxons, a été introduite en Europe comme plante ornementale. Les variétés les plus cultivées seraient «Heavenly blue» à fleurs bleues ; «Pearly gates» à fleurs blanches, «Wedding bells» à fleur lavande ; «Flying saucer» à fleurs bleues et blanches, «Summer skies» et «Blue star» à fleur bleu pâle (37).

Argyreia nervosa (photographie 5).

Les graines noires de Argyreia nervosa ont été utilisées par les Hawaïens comme hallucinogène bon marché, elles sont appelées " wood rose, baby wood rose"(37).

\section{Chimie}

Le pharmacologue Santesson en 1937 publia un rapport concernant les recherches chimiques au sujet de Ipomoea violacea et Rivea corymbosa, mais il ne réussit pas à isoler les principes actifs: Hofmann et Tscherter ont extrait les principes actifs par de l'acétate d'éthyle en milieu alcalin à partir des graines finement broyées. La phase organique est agitée en présence d'acide tartrique, puis à nouveau extraite après passage en milieu alcalin par du bicarbonate. 
Taber et coll. ont extrait les alcaloïdes de la plante cryodesséchée par de l'acétate d'éthyle et de l'éther $(36,38)$ Dans les deux espèces Ipomoea violacea et Rivea corymbosa, on trouve à peu près les mêmes alcaloïdes, qui ont tous pour support le squelette de l'ergoline (figure 2).

Différents alcaloïdes ont été séparés par chromatographie et identifiés par infra rouge :

- l'amide de l'acide d-lysergique (LSA) ou ergine

- l'amide de l'acide d-isolysergique

- la chanoclavine

- le lysergol et l'élymoclavine pour Rivea corymbosa

- l'élymoclavine et l'ergométrine (identifiée plus tard) pour Ipomoea violacea.

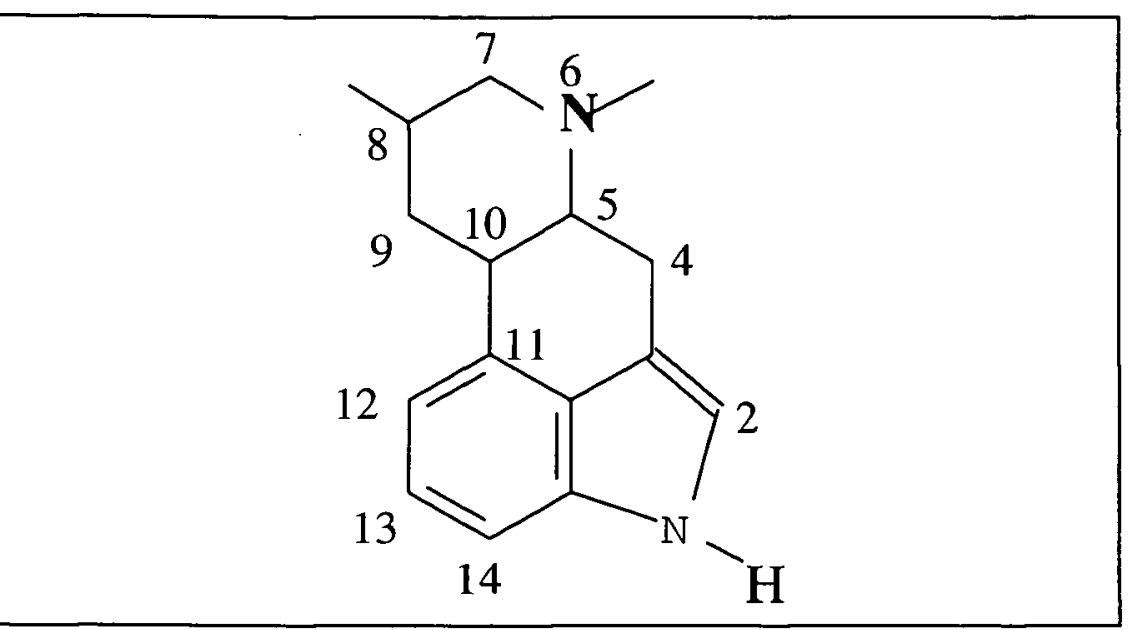

Figure 2 : Formule chimique du noyau ergoline.

\section{Propriétés physico-chimique de l'ergine}

L'ergine ou l'amide de l'acide d-lysergique (LSA) ou Lysergamide ou 9,10-didéhydro-6-méthylergoline-8béta-carboxamide, formule brute : $\mathrm{C}_{16} \mathrm{H}_{17} \mathrm{~N}_{3} \mathrm{O}$; poids moléculaire : 267,$33 ; n^{\circ}$ de CAS : 478-94-4. Elle se décompose à $242^{\circ} \mathrm{C}$

Une graine d'Argyreia nervosa aurait une concentration en ergine équivalente à celles contenues dans 75 à 100 graines d'I. violacea (39).

\section{Effets}

Parmi les différents effets décrits au XVII ${ }^{\text {rne }}$ siècle citons :

«herbe...qui donne une semence nommée ololiuqui. Cette semence trouble et égare les sens, ceux qui en mangent paraissent avoir des visions et assister à des choses terrifiantes».

L'ergine qui aurait à peu près $10 \%$ de l'effet du LSD apparaît être l'alcaloïde majeur. La chanoclavine et l'élymoclavine n'auraient pas d'effets psychotomimétiques (37).

\section{1/Chez l'animal}

Chez la souris, un extrait de I. violacea par voie intra- péritonéale provoque de l'ataxie, des tremblements, une pilo-érection, et une perte de coordination (40).

\section{2/Chez l'homme}

L'ergine, est responsable des effets psychodysleptiques à la dose de $2 \mathrm{mg}$. Les effets sont variables selon le nombre de graines absorbées.

Pour Ipomoea violacea :

Doses faibles : 20 à 50 graines : relaxation, augmentation de la conscience, provoque rarement des hallucinations.

Doses moyennes : 100 à 150 graines : symptômes similaires à ceux produits par l'ingestion de 75 à $150 \mu \mathrm{g}$ de LSD : distorsion spatiale, hallucinations, augmentation des sens et de l'humeur pendant 1 à 4 heures suivie de relaxation et de sommeil.

Doses fortes : 200 à 500 graines : symptômes similaires à ceux produits par l'ingestion de 200 à $500 \mu \mathrm{g}$ de LSD : pupille dilatée, érythème facial, euphorie et augmentation des effets décrits précédemment, mais effets secondaires désagréables : léthargie, nausée, vomissements, douleur abdominale, sensation de froid aux extrémités (37).

Pour Rivea corymbosa :

Cette espèce renfermerait $40 \%$ d'alcaloïdes du type isoergine en moins que Ipomoea violacea. Les effets seraient du même type que ceux énumérés précédemment, mais d'intensité moindre.

\section{Pour Argyreia nervosa :}

Des symptômes adrénergiques seraient prédominants. Un jeune homme de 24 ans se serait intoxiqué par une centaine de graines de Argyreia nervosa. 30 min après la consommation il a vomi. Arrivé aux urgences 1 heure après la consommation des graines, il était agité, les pupilles dilatées, avait de la tachycardie, de l'hypertension et des troubles psychiques. Il a été traité par du charbon activé et du diazépam. 9 heures plus tard, son agitation a cessé, il a pu décrire les visions très colorées qu'il avait vu plus tôt (41).

\section{Mode de consommation}

Les graines sont consommées soit directement par voie orale soit en infusion comme du thé ou bien en macération dans l'éthanol. Ces graines peuvent être commandées sur Internet généralement sous le nom de «Morning Glory» (photographie 6).

Cette substance n'est pas classée dans les produits stupéfiants.

\section{Intoxications}

Peu de cas ont été publiés. L'utilisation de ces graines est relativement limitée du fait des effets secondaires (constipation, léthargie, nausée, vue trouble...) qu'elles provoquent (37). 
Des références d'effets indésirables ont été publiés en 1964 et 1966 (42).

\section{Traitement}

Il faut rassurer le sujet et le surveiller. En principe les symptômes disparaissent en 8 heures. Si nécessaire un peu de diazépam peut être donné pour calmer (37).

\section{Analyse de l'ergine}

Réactions colorées : teinte marron avec le test de Marquis.

Différents systèmes ont été développés par chromatographie sur couche mince, chromatographie liquide haute performance.

Les caractéristiques des maxima des spectres ultra-violet sont de $313 \mathrm{~nm}$ en solution acide et 309 en solution alcaline. Les maxima du spectre infrarouge sont : 1670 , $760,783,1618,1158,1270 \mathrm{~cm}-1$, et les fragments $\mathrm{m} / \mathrm{z}$ caractéristiques du spectre de masse de l'ergine sont 267, 221, 207, 180, 223, 154, 196, 268.

\section{Concentration sanguine - Cas médico- légaux}

Il n'a pas été retrouvé de données concernant les concentrations d'ergine ou des autres alcaloïdes dans les liquides biologiques après consommation de ces Convolvulaceae.

Un suicide aurait été induit par la prise quelques semaines auparavant de graines d'une Convolvulaceae hallucinogène du type Ipomoea $(43,44)$.

\section{Conclusions}

Concernant les consommations des dérivés de l'ergoline, le LSD est de loin le plus utilisé. Il est à ce jour la substance qui présente le plus d'effets hallucinogènes. L'utilisation de chromatographie couplée à des détecteurs de masse en tandem permet d'augmenter la fenêtre de détection de ce produit dans l'urine. Les volubilis ne sont quasiment pas utilisés par les toxicomanes, il semble que les effets secondaires qu'ils induisent les incitent à ne pas renouveler l'expérience.

\section{Remerciements}

Les auteurs remercient Pierre Colas, Carole Paetzold et Nathalie Berquez pour leur contribution aux photographies

\section{Références}

1. Paris R.R., Moyse H. Thallophytes dans Matière Médicale, Tome I, $2^{\text {imc }}$ Edition. Paris : Masson, 1976 : 328-41.

2. Anonyme. LSD Microtablets in Owatonna, Minnesota. Microgram 2003; 4 : 70 .

3. Anonyme. Cocaine/Yohimbine and LSD/Amphetamine mixture in spain. Microgram $2003 ; 12: 277$.

4. Moffat A.-C., Osselton M.-D., Widdop B. Clarke's Analysis of drugs and poisons. The Pharmaceutical Press, 2004, 3 rd Edition. London.

5. Anonyme. Usage et trafic des produits stupéfiants en France en 2002. Document de l'office central pour la répression du trafic illicite des stupéfiants de la Police Nationale Française. 2003.

6. Verstraete A. Statistiques sur l'utilisation de LSD et des hallucinogènes en europe. Toxicorama. $1998 ; 10(2)$ : 5660.

7. Baselt R.-C., Cravey R.-H. Disposition of toxic drugs and chemicals in man. Fourth edition. Foster city : Chemical Toxicology Institute, 1995.

8. Vincent F. Hallucinogènes. In Kintz P., (coordinateur). Toxicologie et Pharmacologie médico-légales, Editions scientifiques et médicales Elsevier, 1998 ; 555-93.

9. Cai J., Henion J. Elucidation of LSD in vitro metabolism by liquid chromatography and capillary electrophoresis coupled with tandem mass spectrometry . J. Anal. Toxicol $1996 ; 20: 27-37$.

10. Verstraete A.-G., Van de Velde E.-J. 2-oxo-3-hydroxyLSD an important metabolite ? Acta Clin. Belgica, 1999 ; sup 1 : 94-6.

11. Peel H.-W., Boynton A.-L. Analysis of LSD in urine using radioimmunoassay, excretion and storage effects. J. Can. Soc. For. Sci. $1980 ; 13$ : 23-8.

12. Bruneton J. Ergolines dans Pharmacognosie. Phytochimie. Plantes médicinales $3^{\text {ine }}$ Edition. Paris : Lavoisier Tec \& Doc : 979-97.

13. Veress T. Study of the extraction of LSD from illicit blotters for HPLC determination. J. Forensic Sci. 1993 ; 38/5:1105-10.

14. Anonyme. Méthodes recommandées pour l'identification du Lysergide (LSD). New-york : Nations Unies, 1990.

15. Harris H.-A., Kane T. A method for identification of Lysergic acid diethylamide (LSD) using a microscope sampling device with Fourier Transform Infrared (FT/IR) spectroscopy. J. Forensic Sci. $1991 ; 36: 1186$.

16. Nelson C., Foltz R.-L. Determination of Lysergic acid diethylamide (LSD), iso-LSD, and N-desmethyl-LSD in body fluids by gas chromatographic/tandem mass spectrometry. Anal. Chem. $1992 ; 64: 1578-85$.

17. Augsburger M., Mangin P. LSD, le phenix des hallucinogènes. Toxicorama, $1998 ; 10(2):$ 61-6.

18. Fixon G., Houdret N., Bernaux F., Imbenotte M., Lhermitte M. Stabilité du LSD dans l'urine. Toxicorama, $1998 ; 10(2): 67-72$.

19. Nakahara Y., Kikura R., Takahashi K. Detection of LSD and metabolite in rat hair and human hair. J. Anal. Toxicol. $1996 ; 20: 323-29$. 
20. Ritter D., Cortese C.-M., Edwards L.-C. Barr J.-L., Chung N.-D., Long C-interference with testing for lysergic acid diethylamide. Clin. Chem. 1997 ; 43 : 635-7.

21. Grobosch T. , Lemm-Ahlers U. Immunoassay screening of lysergic acid and diethylamide (LSD) and its confirmation by HPLC and Fluorescence detection following LSD ImmunElute extraction. J. Anal. Toxicol. 2002 ; $26: 181-6$.

22. Horn C.-K., Klette K.-L., Stout P.-R. LC-MS analysis of 2-oxo-3-hydroxy LSD from urine using a Speedisk ${ }^{\circledR}$ positive-pressure processor with $\operatorname{Cerex}\left({ }^{\circledR}\right.$ PolyChrom TM CLIN II columns. J. Anal. Toxicol. 2003 ; $27-7$ : 459-63.

23. Bergemenn D., Geier A., von Meyer L. Determination of lysergic acid diethylamide in body fluids by high-performance liquid chromatography and fluorescence detection-A more sensitive method suitable for routine use. J. Forensic Sci. 1999 ; 44-2 : 372-79.

24. Blum L.-M., Carenzo E.-F., Rieders F. Determination of lysergic acid diethylamide (LSD) in urine by instrumental high-performance thin layer chromatography. J. Anal. Toxicol. $1990 ; 14: 285-7$.

25. Sauvage M.-F., Marquet P., Ragot S., Lachâtre F. Dupuy J.-L., Lachâtre G. Dosage du LSD et de trois de ses métabolites ou isomères dans le sérum et le sang total par LCES-MS. Toxicorama, $1998 ; 10(2): 73-9$.

26. Poch G.-K., Klette K.-L., Anderson C. The quantitation of 2-oxo-3-hydroxy-Lysergic acid diethylamine (O-HLSD) in human urine specimens, a metabolite of LSD : Comparative analysis using liquid chromatography/selected ion monitoring mass spectrometry and liquid chromatography/ion trap mass spectrometry. J. Anal. Toxicol. $2000 ; 24: 170-9$.

27.Paul B.-D., Mitchell J.-M., Burbage R., Moy M., Sroka R. Gas chromatographic-electron impact mass fragmentometric determination of lysergic acid diethylamide in urine. J. Chromatogr. 1990 ; 529 : 103-12.

28. Reuschel S.-A., Percey S.-E., Liu S., Eades D.-M., Foltz R.-L. Quantitative determination of LSD and a major metabolite 2-oxo-3-hydroxy-LSD, in human urine by solid phase extraction and gas chromatography tandem mass spectrometry. J. Anal. Toxicol.1999 ; 23 : 306-12.
29. Klock J.-C. Boerner U., Becker C.E. Coma, hyperthermia and bleeding associated with massive LSD overdose : a report of eight cases. West J. Med. 1974, 102, 183-88.

30. Griggs E.-A, Ward M. LSD toxicity : a suspected cause of death. J. K. Med. Assoc. 1977 ; 172-3.

31. Fysh R.-R., Oon M.-CH., Robinson K.-N., Smith R.-N., White P.-C., Whitehouse M.-J. A fatal poisoning with LSD. Forensic Sci. Int. 1985 ; 28 : 109-13.

32. Smith R.-N., Robinson K. Body fluid levels of lysergide (LSD). Forensic Sci. Int. 1985 ; 28 : 229-37.

33. Hofmann A. Le Teonacalt et l'ololiuqui, deux anciennes drogues magiques du Mexique. Bulletin des stupéfiants. $1971 ; 7-14$.

34. Pelt J.-M. Drogues et plantes magiques. Paris : Horizons de France, 1971.

35. Schultes R.-E., Hofmann A. The botany and chemistry of hallucinogens. Thomas book 1973.

36. Robert M.-P. Ololiuqui : Rivea corymbosa, Ipomoea violacea, drogue magique des aztèques. Thèse pour le diplôme d'état de docteur en pharmacie, Lille, 1988.

37. Ellenhorn M.-J., Barceloux D.-G. Psychoactive and hallucinogenic plant. In Medical Toxicology. Diagnostic and treatment of human poisoning. New York Elsevier. $1988 ; 1288-90$.

38. Taber W.-A., Heacock R.-A., Mahon M.-E. Ergot-type alkaloïds in vegetative tissues of Rivea Corymbosa. Phytochemistry, $1963 ; 2: 99-101$.

39. Brown J.-K., Malon M.-H. "Legal-High" constituants, activity, toxicology of herbal folklore. Clin. Toxicol. $1978 ; 12: 1-31$.

40. Rice W.-B., Genest K. Acute toxicity of extract of morning glory seeds in mice. Nature $1965 ; 207: 302-3$.

41. Furbee R.-B., Curry S.-C., Kunkle D.-B. Ingestion of Argyreia nervosa (Hawaiian baby woodrose) seeds. Vet. Human Toxicol. $1991 ; 33-4: 370$.

42. Reynolds J.-E.-F. Martindale. The Extra Pharmacopoeia. The pharmaceutical press, $1993: 1395$.

43. Bruneton J. Plantes toxiques. Végétaux dangereux pour l'homme et les animaux. Lavoisier Tec \& Doc. $2^{\text {inc }}$ édition. $1996 ; 11$

44. Cohen S. Suicide following morning glory seed ingestion. Am J. Psychiatry, 1964 ; 120, 1024-5. 